\title{
Diabetes remission off medications is not a suitable endpoint for comparing bariatric/metabolic surgery with pharmacotherapy. Reply to Halpern B, Cercato C, Mancini MC [letter]
}

\author{
David E. Cummings ${ }^{1}$
}

Received: 23 May 2016 / Accepted: 31 May 2016/Published online: 7 July 2016

(C) Springer-Verlag Berlin Heidelberg 2016

Keywords Bariatric surgery · Biliopancreatic diversion · Diabetes · Diabetes surgery summit · Gastric bypass · Metabolic surgery $\cdot$ Randomised clinical trial $\cdot$ Randomised controlled trial $\cdot$ Randomised trial $\cdot$ Sleeve gastrectomy

$\begin{array}{ll}\begin{array}{l}\text { Abbreviations } \\ \text { CROSSROADS }\end{array} & \begin{array}{l}\text { Calorie Reduction Or Surgery: Seeking to } \\ \text { Reduce Obesity And Diabetes Study }\end{array} \\ \text { MLI } & \begin{array}{l}\text { Medical/lifestyle intervention } \\ \text { RCT }\end{array} \\ \text { Randomised clinical trial }\end{array}$

To the Editor: On behalf of the authors of the Calorie Reduction Or Surgery: Seeking to Reduce Obesity And Diabetes Study (CROSSROADS) [1], I sincerely thank Halpern et al for their thoughtful critique of our trial [2].

Among other things, these investigators challenge the use of diabetes remission as the primary endpoint in any randomised clinical trial (RCT) comparing bariatric/ metabolic surgery vs a medical/lifestyle intervention (MLI). 'Diabetes remission' is defined in various RCTs as achievement of an $\mathrm{HbA}_{1 \mathrm{c}}$ target - typically $<6.0 \%$ (42 $\left.\mathrm{mmol} / \mathrm{mol}\right)$, $<6.5 \%$ (48 $\mathrm{mmol} / \mathrm{mol})$ or $<7.0 \%(53 \mathrm{mmol} / \mathrm{mol})$ _off all diabetes medications. Since the most powerful tool to attain that goal in MLI groups is the use of medications, Halpern et al feel it is unfair to require those study participants to be off such agents in order to achieve the primary outcome. This is a very

David E. Cummings

davidec@u.washington.edu

1 Department of Medicine, University of Washington, Box 358280 (mail stop 111), Seattle, WA 98195, USA reasonable critique: the topic of extensive discussion at the recent 2nd Diabetes Surgery Summit, a consensusdevelopment conference that generated new guidelines for the use and study of bariatric/metabolic surgery to treat type 2 diabetes [3]. Although the primary endpoint of the CROSSROADS trial was, indeed, diabetes remission [1], during the time since the study was initially designed several years ago we have generally come to agree with the assertion that in surgery-vs-MLI RCTs in this domain, measurement of glycaemic control-i.e. achievement of a specified $\mathrm{HbA}_{1 \mathrm{c}}$ threshold with or without diabetes medications - is a better primary outcome than diabetes remission in most cases.

It is important to note, however, that medications are not the only instruments that patients randomised into non-surgical treatment arms in such studies can use to improve their diabetes, and the lifestyle intervention in CROSSROADS was particularly intensive, probably more so than in any related RCT to date. Modelled after the Look AHEAD (Action for Health in Diabetes) trial, this intervention included $\geq 45 \mathrm{~min} /$ day of aerobic exercise, $\geq 5$ days/week, largely under the direct supervision of a personal trainer in a well-equipped, dedicated research gym. It also involved a closely supervised weight-reducing and glucoselowering diet, with in-person one-on-one and weekly group counselling sessions. Although this rigorous lifestyle intervention would be difficult to implement in clinical practice, a major goal of CROSSROADS was to determine whether, under the best of circumstances, an aggressive diet, exercise and state-ofthe-art medical regimen can match the impact of gastric bypass on diabetes status. The answer in this case was 'no'.

Importantly, our findings are consistent with those of ten other published RCTs to date directly comparing several bariatric/metabolic operations against a variety of MLIs to treat type 2 diabetes (Fig. 1) [3]. A universal finding among these trials is that surgery is superior to MLIs for all glycaemic outcomes. This includes not just the possibly unfair endpoint 


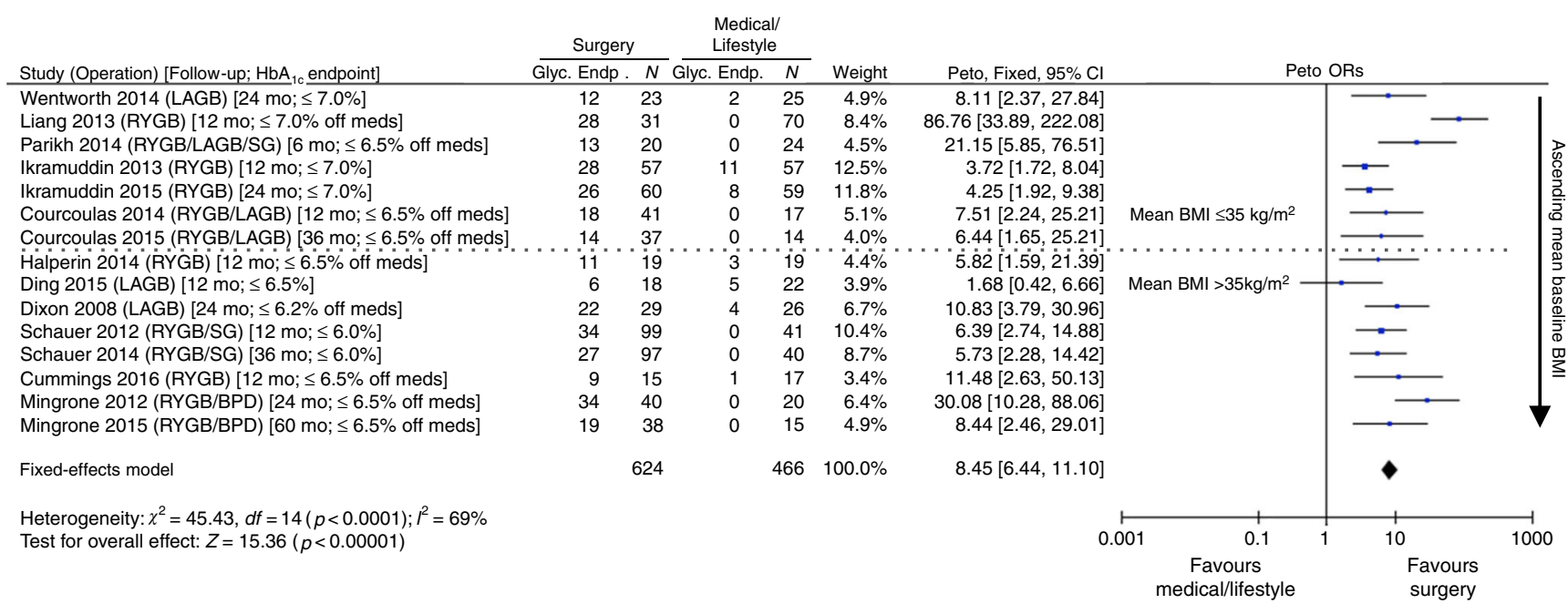

Fig. 1 Forest plot of Peto ORs of main glycemic endpoints (Glyc. Endp.), as defined in each trial, from published RCTs of bariatric/metabolic surgery compared with medical/lifestyle treatments for diabetes. Data are arranged in order of ascending mean baseline BMI; the dotted line separates trials performed with cohorts exhibiting an average baseline BMI $\leq 35 \mathrm{~kg} / \mathrm{m}^{2}$ and $>35 \mathrm{~kg} / \mathrm{m}^{2}$. Study duration and $\mathrm{HbA}_{1 \mathrm{c}}$ endpoint thresholds are shown in brackets in column 1 , where 'off meds' indicates a threshold achieved off all diabetes medications; otherwise, endpoints represent $\mathrm{HbA}_{1 \mathrm{c}}$ thresholds achieved with or without such medications.
ORs $>1$ indicate a positive effect of surgery compared with medical/ lifestyle treatment. For each study, the OR is shown with its $95 \%$ CI. The pooled Peto ORs $(95 \% \mathrm{CI})$ for all data were calculated under the assumption of a fixed-effects model. BPD, biliopancreatic diversion; LAGB, laparoscopic adjustable gastric banding; RYGB, Roux-en-Y gastric bypass; SG, sleeve gastrectomy. For full details of the references cited in the figure, please see [3]. Reprinted with permission of the American Diabetes Association, Inc., Copyright 2016 [3] of diabetes remission, but also glycaemic control, diabetes improvement and diabetes medication use.

Halpern et al also correctly point out that CROSSROADS was not large enough to examine endpoints in subgroups with a baseline BMI $<35 \mathrm{~kg} / \mathrm{m}^{2}$ vs $\geq 35 \mathrm{~kg} / \mathrm{m}^{2}$, the traditional threshold for use of bariatric/metabolic surgery in patients with type 2 diabetes, nor to analyse the impact of baseline BMI on glycaemic endpoints. We stated in the paper that the trial was not powered for such analyses, which were therefore merely exploratory. In hindsight, however, it might have been best not to comment on these matters at all.

Nevertheless, there is now ample evidence beyond CROSSROADS to address the question of whether the antidiabetes benefits of bariatric/metabolic surgery are attenuated in patients with a preoperative BMI $<35 \mathrm{~kg} / \mathrm{m}^{2}$ [4]. Intuitively, one might predict this because leaner people lose less body weight after surgery than do the severely obese. However, most bariatric/metabolic operations engage weight-independent antidiabetes mechanisms, which are presumably equally active in patients with a lower BMI [5]. Empirically, in a recent comprehensive meta-analysis of all studies reporting diabetes remission rates after bariatric/metabolic surgery, describing 94,579 surgical patients with type 2 diabetes, the overall remission rate was equivalent among the 60 studies in which mean preoperative BMI was $\geq 35 \mathrm{~kg} / \mathrm{m}^{2}$ compared with the 34 studies in which mean preoperative BMI was $<35 \mathrm{~kg} / \mathrm{m}^{2}$ (71\% vs $72 \%$, respectively) [6]. Although the sheer size of this systematic review confers validity, it did not control for the quality of evidence in each study. Nevertheless, the results resonate with data from arguably the highest quality trial in the field: the large, long-term Surgical Treatment and Medications Potentially Eradicate Diabetes Efficiently (STAMPEDE) RCT [7]. At all time points over the course of 3 years in this study, surgical patients displayed far greater $\mathrm{HbA}_{1 \mathrm{c}}$ lowering (despite lesser diabetes medication usage) compared with non-surgical patients, and these results were identical in subgroups with average baseline $\mathrm{BMI}<35 \mathrm{~kg} / \mathrm{m}^{2} \mathrm{vs} \geq 35 \mathrm{~kg} / \mathrm{m}^{2}$. Extending this Level-1 evidence to Level-1A evidence (meta-analysis of RCTs), Fig. 1 shows that among the 11 relevant RCTs conducted to date, including 1090 randomised participants, the magnitude of surgical superiority over MLIs for diabetes remission and/or glycaemic control was similar among the trials wherein the average baseline BMI of the study cohort was $<35 \mathrm{~kg} / \mathrm{m}^{2}$ and $\geq 35 \mathrm{~kg} / \mathrm{m}^{2}[3,4]$.

The very long-term, exemplary Swedish Obese Subjects (SOS) study [8] (which Halpern et al justifiably cite), as well as other investigations, found that baseline BMI did not predict surgical benefits on diabetes prevention, remission or recurrence after initial remission, or the magnitude of its effects on heart attacks, strokes, cancer or death. In contrast, high levels of baseline fasting insulin and/or glucose (presumably reflecting insulin resistance) do predict the benefits of surgery on most of these endpoints [8]. This strongly suggests that the advantages of bariatric/metabolic surgery regarding clinically relevant hard outcomes are more related to its impact on glucose homeostasis than body weight per se, seriously challenging the longstanding use of BMI as the primary criterion for surgical selection. 
Of course, surgical benefits must be balanced against risks, and Halpern et al appropriately highlight that bariatric/ metabolic surgery can cause complications. The question is whether these risks are outweighed by the hazards suffered among non-surgical patients, who typically achieve lesser degrees of weight loss, diabetes improvement and reductions of other cardiovascular and cancer risks. Nine studies have now uniformly demonstrated that, at least among people qualifying for bariatric surgery by traditional standards, long-term allcause mortality is reduced among those who undergo surgery compared with those who do not [9]. Importantly, these are all non-randomised studies, subject to potential biases from unmeasured confounding. Perhaps individuals who elect to undergo an aggressive surgical strategy are more motivated to engage in other health-conscious behaviours. Hence, we heartily agree with Halpern et al regarding the need for long-term safety and efficacy data from RCTs comparing surgical vs non-surgical approaches to treat type 2 diabetes. Towards that end, CROSSROADS has now merged its study participants with those in three related RCTs to form a unified, long-term RCT - the Alliance of Randomised trials of Medicine vs Metabolic Surgery in Type 2 Diabetes (ARMMS-T2D) consortium - which will hopefully help redress this knowledge gap, including among patients with a preoperative BMI $<35 \mathrm{~kg} / \mathrm{m}^{2}$.

Funding The CROSSROADS trial was funded by NIH (NIDDK) grant DK089528.

Duality of interest The author declares that there is no duality of interest associated with this paper.

Contribution statement The author was the sole contributor to this paper.

\section{References}

1. Cummings DE, Arterburn DE, Westbrook EO et al (2016) Gastric bypass surgery vs intensive lifestyle and medical intervention for type 2 diabetes: the CROSSROADS randomised controlled trial. Diabetologia 59:945-953

2. Halpern B, Cercato C, Mancini MC (2016) Diabetes remission off medications is not a suitable endpoint for comparing bariatric/metabolic surgery with pharmacotherapy. Diabetologia. doi:10.1007/s00125-0164007-3

3. Rubino F, Nathan DM, Eckel RH et al (2016) Metabolic surgery in the treatment algorithm for type 2 diabetes: a joint statement by international diabetes organizations. Diabetes Care 39:861-877

4. Cummings DE, Cohen RV (2016) Bariatric/metabolic surgery to treat type 2 diabetes in patients with a BMI $<35 \mathrm{~kg} / \mathrm{m}^{2}$. Diabetes Care 39:924-933

5. Batterham RL, Cummings DE (2016) Mechanisms of diabetes improvement following bariatric/metabolic surgery. Diabetes Care 39: 893-901

6. Panunzi S, De Gaetano A, Carnicelli A, Mingrone G (2015) Predictors of remission of diabetes mellitus in severely obese individuals undergoing bariatric surgery: do BMI or procedure choice matter? A meta-analysis. Ann Surg 261:459-467

7. Schauer PR, Bhatt DL, Kirwan JP et al (2014) Bariatric surgery versus intensive medical therapy for diabetes - 3-year outcomes. N Engl J Med 370:2002-2013

8. Sjöstrom L (2013) Review of the key results from the Swedish Obese Subjects (SOS) trial - a prospective controlled intervention study of bariatric surgery. J Intern Med 273:219-234

9. Schauer PR, Mingrone G, Ikramuddin S, Wolfe B (2016) Clinical outcomes of metabolic surgery: Efficacy of glycemic control, weight loss, remission of disease, and complications. Diabetes Care 39:902911 